REVIEW

\title{
Non-invasive brain stimulation for Parkinson's disease: a systematic review and meta-analysis of the literature
}

\author{
F Fregni, D K Simon, A Wu, A Pascual-Leone
}

J Neurol Neurosurg Psychiatry 2005;76:1614-1623. doi: 10.1136/jnnp.2005.069849

A systematic review and meta-analysis were conducted to quantify the efficacy of transcranial magnetic stimulation (TMS) and electroconvulsive therapy (ECT) for the treatment of motor dysfunction in patients with Parkinson's disease (PD). Prospective studies which evaluated the effects of either TMS (12 studies) or ECT (five studies) on motor function in PD using the motor subscale of the Unified Parkinson's Disease Rating Scale (UPDRS) for TMS studies and any continuous measures of motor function in PD for ECT studies were included. The pooled effect size (standardised mean difference between pre-treatment versus post-treatment means) from a random effects model was 0.62 (95\% confidence interval: $0.38,0.85)$ for TMS treatment and $1.68(0.79,2.56)$ for ECT treatment, and from a fixed effects model was $0.59(0.39,0.78)$ for TMS treatment and $1.55(1.07,2.03)$ for ECT treatment. TMS, across applied stimulation sites and parameters, can exert a significant, albeit modest, positive effect on the motor function of patients with PD. ECT also may exert a significant effect on motor function in PD patients.

See end of article for authors' affiliations

\section{Correspondence to:} Felipe Fregni, 330 Brookline Ave, KS 452, Boston, MA 02215, USA ffregni@bidmc.harvard. edu

Received 17 April 2005 Revised version received 13 June 2005 Accepted 14 June 2005
$\mathrm{P}$ arkinson's disease (PD) is a progressive neurodegenerative disease that predominantly affects elderly people, and thus it tends to be more prevalent as the population ages. Although the motor symptoms of PD are mainly treated with drugs, the clinical utility of these medications tends to become limited over the years, often due to adverse effects such as dyskinesias (for review, see Olanow et al ${ }^{1}$ ). Nonpharmacological approaches, such as deep brain stimulation (DBS), are effective in the treatment of PD motor symptoms in selected patients. For instance, in a meta-analysis, Boucai et al showed that functional neurosurgery for PD is effective for improving motor function and dyskinesias, ${ }^{2}$ and may even offer advantages over pharmacological approaches. Although recent developments in invasive brain stimulation for PD, such as improvement in the DBS technique and minimally invasive cortical stimulation, have reduced the surgical risks, neurosurgical procedures are still costly and invasive. Therefore, non-invasive forms of brain stimulation are desirable. Electroconvulsive therapy (ECT) and repetitive transcranial magnetic stimulation (rTMS), both types of non-invasive brain stimulation, have been used in PD patients and have been suggested as possible therapeutic tools.
rTMS is a non-invasive, well tolerated technique of brain stimulation based on electromagnetic induction. The effects of rTMS spread from the directly targeted brain region along specific neural connections to distant cortical and subcortical regions. ${ }^{3}{ }^{4}$ Therefore, it is possible by using rTMS to modulate activity in specific neural networks, using cortical targets as "entry ports". Several studies have investigated the use of rTMS to treat the motor symptoms of PD patients. The results of these trials are mixed and no conclusion has been reached so far. ECT induces current in the brain by direct transcranial application of a strong current pulse and is associated with the induction of a seizure. The mechanisms of action of ECT are unclear, but several studies have reported that ECT is effective for treating PD patients. However, most of these studies are case reports, and thus, no conclusions have been reached about the utility of ECT in patients with PD.

Therefore, whether non-invasive brain stimulation (ECT or TMS) is effective for treating PD remains unclear; such information would be important to either support or provide evidence against future larger trials of non-invasive brain stimulation for PD. Here, we systematically review studies that examined the effects of TMS or ECT on motor function in PD. We critically assess the heterogeneity of these study results to better understand the factors that may contribute to a better motor outcome following non-invasive brain stimulation.

\section{METHODS}

\section{Literature search}

The first step of our meta-analysis was a selective literature search for articles published from 1980 to January 2005. We used the following databases: MEDLINE, EMBASE, Cochrane, and SCIELO. In addition, we examined reference lists in systematic reviews and retrieved papers, searched conference abstracts, and talked to clinical experts. To check for unpublished trials, we contacted experts in the field, consulted the CRISP database, and searched for abstracts. Our key search terms were "Parkinson's disease", "transcranial magnetic stimulation", "electroconvulsive therapy", "brain stimulation", and "noninvasive brain stimulation". This strategy

\footnotetext{
Abbreviations: DBS, deep brain stimulation; ECT, electroconvulsive therapy; PD, Parkinson's disease; rTMS, repetitive transcranial magnetic stimulation; $S D$, standard deviation; TMS, transcranial magnetic stimulation; UPDRS, Unified Parkinson's Disease Rating Scale; $95 \% \mathrm{Cl}$, $95 \%$ confidence interval
} 
yielded 127 studies for TMS and PD, and 143 studies for ECT and PD. Using the terms "Parkinson's disease" with either "brain stimulation" or "noninvasive brain stimulation" yielded no additional studies.

\section{Selection criteria}

We included prospective studies that evaluated the effects of either TMS or ECT on motor function in PD. We adopted the following inclusion criteria: (i) manuscript written in English, German, Italian, French, Spanish, or Portuguese; (ii) use of TMS or ECT in PD patients; and (iii) motor effects measured using the motor section of the Unified Parkinson's Disease Rating Scale (UPDRS) for TMS studies or any other continuous clinical scale that evaluated the motor symptoms of PD for ECT studies; we decided to allow other clinical scales, rather than only the UPDRS (for example, the Webster scale was used in the studies by Fall ${ }^{5}$ and Andersen ${ }^{6}$ ) for ECT studies, as most of them were carried out at the beginning of the 1980s and therefore did not report UPDRS scores; (iv) the report had to have been published in a book, journal, proceeding, or indexed abstracts; and (v) the study had to report the mean and standard deviation (SD) of the motor function before and after treatment or provide other statistical parameters that could be used to deduce these values. For studies that met our criteria but did not report these scores, the authors were contacted to provide these data if available. Four out of five consulted authors replied to our request, and three of these four could provide data. For cases where two or more published studies reported overlapping data sets, we chose the study with the largest population. Case reports or series of case reports were excluded.

\section{Extraction of the outcome measures}

The data were collected using a semi-structured form for each study by one of the authors and checked by another investigator. Discrepancies were resolved by consensus and a third author consulted if necessary. The following variables were extracted: (i) mean and SD of the motor section (part III) of the UPDRS (or other clinical scale for the ECT studies) for baseline and after treatment for the active and placebo group (if the study was sham controlled); (ii) mean and SD for the follow up period evaluation (if these data were available); (iii) study design; (iv) demographic and clinical characteristics (for example, number of patients, age, gender, disease duration); (v) baseline motor UPDRS and Hoehn and Yahr stage; (vi) TMS parameters (frequency, intensity, number of pulses, number of sessions); and (vii) ECT parameters (uni/bilateral, intensity, number of sessions).

For the studies with more than one active group (that is, two different doses of TMS), we considered each group as one study in the quantitative analysis. This approach was used for the following three studies: Mally et al (four different doses of TMS), de Groot et al ${ }^{8}$ (two different doses of TMS) and Lefaucheur et al (two different doses of TMS).

\section{Systematic review}

Because the literature on ECT and TMS in PD consists mainly of uncontrolled studies, we included both controlled and uncontrolled studies, and compared the results of the two sets of studies.

\section{Qualitative analysis}

We first assessed sources of heterogeneity across studies. Major features contributing to between-study heterogeneity were determined a priori and evaluated in our analysis, and included study design (controlled and uncontrolled studies), PD clinical characteristics (motor disability as indicated by baseline motor UPDRS and baseline Hoehn and Yahr stage, and duration of disease), demographic characteristics (age, gender), and treatment characteristics (TMS and ECT parameters). Although analyses of subsections of the motor UPDRS, such as tremor, rigidity, gait, and bradykinesia, would have provided useful information, these data were not available in most of the selected studies.

\section{Quantitative analysis}

All our analyses were performed using Stata statistical software, version 8.0 (StataCorp, College Station, TX). For the continuous measures of motor function, we calculated the standardised mean difference (Cohen d) based either on the pre- and post-test values of one group within each study or comparison of the mean changes in pre-treatment to posttreatment UPDRS of the two independent subject groups (sham and active rTMS) in the controlled trials using the means and SDs, or estimated from the graphs (study by Ikeguchi $\left.e t a l^{10}\right)$. For the post-treatment value, we used the evaluation that was carried out immediately after the treatment. However, for the trials that also reported an additional post-treatment evaluation within 2 months of the end of treatment (most of them reported a 30 day follow up after the end of treatment), we conducted a separate analysis to evaluate the long term effects of this treatment comparing it to the baseline value (pre-treatment). In the next step, we measured the pooled weighted effect size using random and fixed effects models. The random effect model gives relatively more weight to smaller studies and wider confidence intervals than the fixed effect model and its use has been advocated if there is heterogeneity between studies. ${ }^{11}$ Although the test for heterogeneity failed to detect heterogeneity in one of our analyses, we decided to report both values (from random and fixed effects models). As all rTMS trials reported results using the motor UPDRS, we also reported the weighted pooled mean difference to facilitate interpretation of the results.

Heterogeneity was evaluated with the Q statistic. Although some of these tests disclosed a non-significant heterogeneity, this test may have been underpowered due to the small number of studies; therefore, we synthesised the results from individual studies by using the DerSimonian and Laird random effects model to incorporate both within and between study variability and the fixed effect models to compare the results.

As our meta-analysis included small studies and these studies usually have large effect sizes, we evaluated the influence of individual studies, computing the meta-analysis estimates and omitting one study at a time.

As we expected heterogeneity in the effect of treatment between studies, we assessed this source of heterogeneity, in an exploratory manner, performing a meta-regression in which the outcome was the effect size and the covariates were the variables that could have influenced the effect size, such as study design, demographic and clinical characteristics, and TMS parameters. Medication use was not included in this analysis because these data are unavailable for most of these studies. This analysis was not performed for the ECT analysis as only five small studies were included.

We assessed publication bias using the Begg modified funnel plot, ${ }^{12}$ in which the standardised mean difference from each plot was plotted against the standard error.

\section{RESULTS}

\section{Studies retrieval}

Using the words "transcranial magnetic stimulation" and "Parkinson's disease", we found 127 citations. Five additional citations were found by searching the bibliographies of the retrieved papers and reviews. Therefore, 132 publications were identified and carefully reviewed. Initially, we excluded 110 references for the following reasons: TMS was used to measure other neurophysiological parameters, or the 

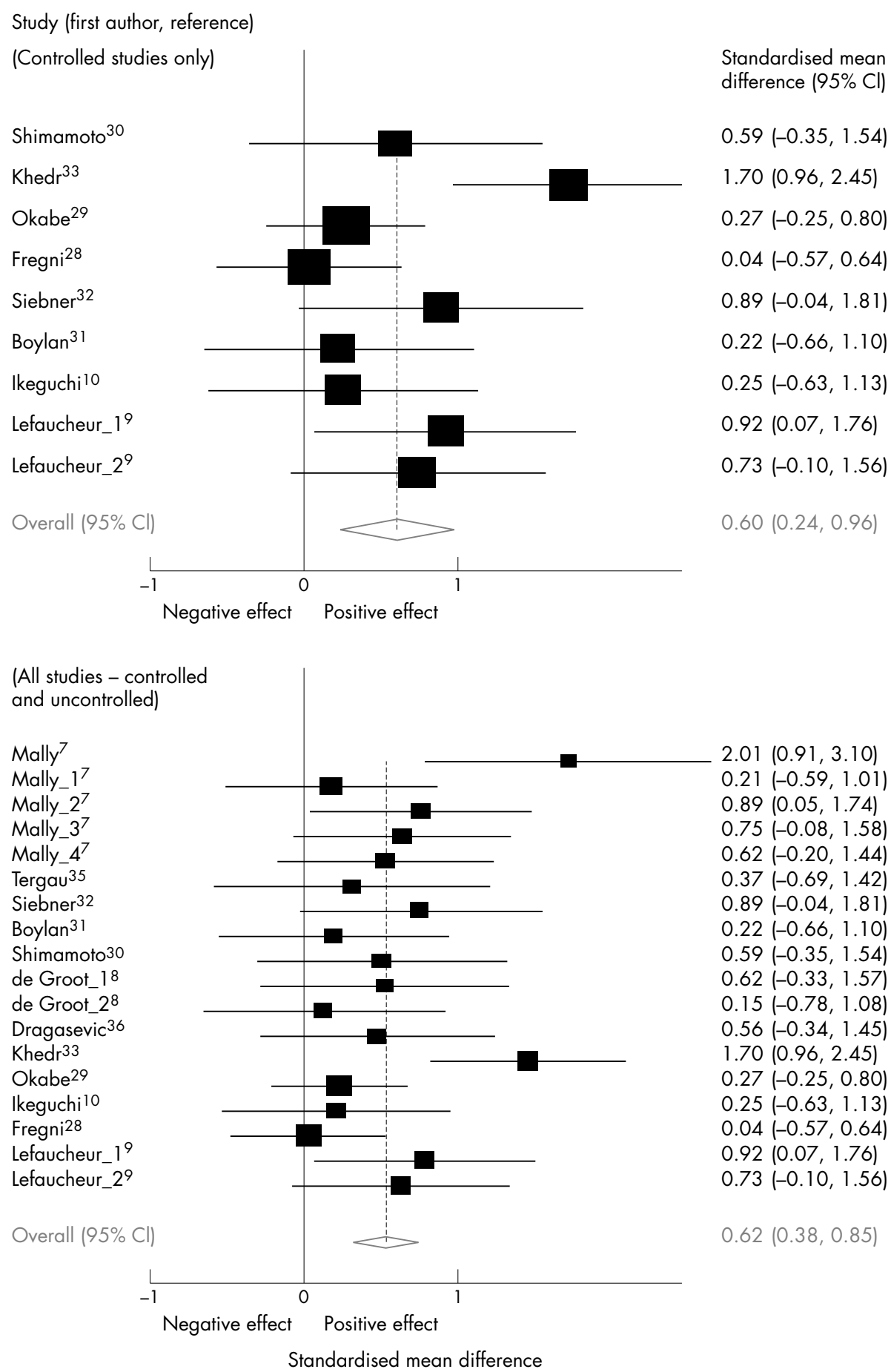

Figure 1 Effect sizes (standardised mean difference in motor UPDRS scores from baseline to immediately after treatment) from the random effects model for the sham controlled studies only (at the top) and for all TMS studies (controlled and uncontrolled) (at the bottom). A positive effect indicates an improvement in motor function. Effect sizes are Cohen d (standardised mean difference), error bars represent the $95 \%$ confidence interval.

publications were reviews or case reports, dealt with other topics, or were in another language. Of the remaining 22 references, 10 were excluded because they used endpoints other than UPDRS to measure improvement in motor function, for instance in reaction time, ${ }^{13-17}$ motor evoked potential/silent period, ${ }^{18} 19$ and speed of movement, ${ }^{19}{ }^{20}$ or had poor data reporting (the mean and SD for the baseline and post-treatment evaluation did not agree with the $\mathrm{p}$ value provided in the study table $\left.{ }^{21}\right)$. Thus 12 studies were selected for the final analysis, of which eight were placebo controlled studies and four uncontrolled studies.

The same process was performed for ECT. Using the words "electroconvulsive therapy" and "Parkinson's disease", we found 143 citations. Three additional citations were found by searching the bibliographies of the retrieved papers and reviews. Of the 146 publications identified, we excluded 135 for the following reasons: they were reviews or case reports, dealt with other topics, or were in another language. Of the 


\begin{tabular}{lll} 
Table 1 & Demographic findings \\
& TMS & \\
\hline & ECT \\
\hline Number of patients & 224 & 49 \\
Age (years) & $63.0 \pm 3.8$ & $68.6 \pm 3.6$ \\
Sex (M:F ratio) & $1.3: 1$ & $1.1: 1$ \\
Disease duration (years) & $6.6 \pm 3.1$ & $13.8 \pm 2.8$ \\
Baseline UPDRS & $25.7 \pm 8.8$ & $-^{*}$ \\
Baseline HY & $2.4 \pm 0.8$ & $3.6 \dagger$ \\
\hline
\end{tabular}

Values are mean \pm SD. HY, Hoehn and Yahr stage.

*Not reported in the ECT trials; treported in just one ECT trial.

remaining 11 references, six were excluded because they were case reports, ${ }^{22-24}$ did not provide numbers regarding motor outcome, ${ }^{25}{ }^{26}$ or were duplicated studies. ${ }^{27}$ Therefore five studies were selected for the final analysis.

The demographic findings of these studies are summarised in table 1 .

\section{Meta-analysis results for TMS trials}

Characteristics of the TMS trials are summarised in table 2 . Initially, we combined data from the controlled, double blind studies only. Pooling the data of the eight controlled trials, we found a pooled effect size (standardised mean difference between before and after TMS application) from the random effects model of 0.60 (95\% confidence interval (95\% CI): 0.24 , 0.96 ) and from the fixed effects model of 0.56 (95\% CI: 0.30 , 0.81 ) (fig 1). The test for heterogeneity failed to show a significant heterogeneity (Q8, $\left.\chi^{2}=15.4, \mathrm{p}=0.052\right)$. These results are similar to the pooled effect size when all studies are included (rather than just double blind studies): the pooled weighted effect size from the random effects model was $0.62(95 \%$ CI: $0.38,0.85)$ and from the fixed effects model was 0.59 (95\% CI: 0.39, 0.78); no significant heterogeneity was found (Q17, 24.45, p=0.11) (fig 1). This result indicates that the inclusion of uncontrolled studies into our meta-analysis did not alter the outcome of our analysis.

As patients with PD can experience a strong placebo effect, we analysed the effect size on UPDRS change (comparison between before and after treatment) in the sham rTMS group. The method of rTMS placebo varied across the different studies, for example, sham coil, ${ }^{98-30}$ active coil angled at either $45^{\circ 31} 32$ or $90^{\circ, 33}$ and active coil stimulation of occipital area, ${ }^{10}$ and are not expected to cause motor function improvement other than perhaps through a placebo effect. For the studies that used active and sham control groups, such as that by Okabe et al, ${ }^{29}$ we used the data from the sham control group. This analysis disclosed that there was a small placebo effect which was not significant. The pooled weighted effect size from the random effects model was 0.1 (95\% CI: $-0.16,0.35)$. The fixed effects model yielded almost the same value as the random effects model, since the test for heterogeneity showed that these data are strongly homogeneous (Q7, $\chi^{2}=1,14, p=0.992$ ) (fig 2).

In order to check whether the effects shown by the TMS studies were significant when compared to the placebo group, we calculated the effect size using the changes between pre- and post-treatment mean UPDRS scores for the active versus sham TMS groups. This analysis showed a pooled effect size from the random effects model of $1.19(95 \%$ CI: $0.44,1.94)$ and from the fixed effects model of 0.87 (95\% CI: $0.59,1.15)$. The test for heterogeneity confirmed that there was a significant heterogeneity in this analysis (Q7, $\left.\chi^{2}=45.61, \mathrm{p}<0.0001\right)$. This finding demonstrates that the motor improvement observed in the active group cannot be explained by a placebo effect only (fig 3 ).

In order to provide a more meaningful clinical result, we calculated the pooled weighted mean difference in the motor UPDRS scores (difference of the means between before and after treatment). Following this analysis, the pooled weighted mean difference was 5.89 (95\% CI: 3.36, 8.43) for the random effects model and 4.14 (95\% CI: 2.78, 5.50) for the fixed effects model (table 3 ).

We performed a meta-regression analysis in which we evaluated the following covariates: year of study, study design, age, disease duration, baseline Hoehn and Yahr stage, frequency of stimulation, number of TMS pulses per session, intensity of TMS, and number of sessions. Although we performed multiple testing for this analysis, we considered these to be exploratory analyses and so did not correct for multiple comparisons. The meta-regression would not support the inclusion of all variables at the same time given the small number of studies and patients. These analyses showed that none of these variables could explain the source of the variability across the different studies (table 4 ).

As some studies evaluated the long lasting effects of rTMS, we analysed this effect comparing the motor function scores 30 days after the completion of treatment against baseline motor function. Six studies performed follow up evaluation; three were controlled and the other three were uncontrolled trials. Follow up evaluation was carried 30 days after the end of treatment, except for the study of Fregni et a ${ }^{28}$ which evaluated patients 2 months after treatment. The pooled weighted effect size (comparing motor function at follow up versus baseline) from the random effects model was 0.71 (95\% CI: $0.26,1.17)$ and from the fixed effects model was 0.59 (95\% CI: 0.34, 0.84). The test for heterogeneity confirmed that there was a significant heterogeneity in this analysis $\left(\mathrm{Q} 8, \chi^{2}=25.21, \mathrm{p}=0.001\right)$. Interestingly, the studies that showed a significant long lasting effect were those that showed a significant effect of TMS on motor function immediately after treatment, ${ }^{73} 34{ }^{36}$ whereas the other two studies $^{28} 29$ did not show significant motor change either immediately after TMS or at the follow up. This finding suggests that an immediate motor benefit after TMS, when present, is predictive of a long lasting effect (fig 2).

We evaluated the influence of individual studies by computing the meta-analysis estimates and omitting one study at a time. Figure 4 shows the results of the random effects estimates excluding one study at a time. The two studies which had the largest individual influence were the studies of Fregni et $a l^{28}$ and Khedr et al. ${ }^{33}$ Interestingly, each study had the opposite influence: whereas exclusion of Fregni's study increases the overall estimate $(0.66,95 \%$ CI: $0.43,0.90)$, exclusion of Khedr's study decreases the overall estimate (0.51, 95\% CI: 0.31, 0.71). However, the overall finding of a positive effect of TMS on motor function in PD remains significant after the exclusion of any single study.

In order to test for publication bias, we used the funnel plot for visual assessment. The funnel plot is helpful to identify whether the results are biased due to exclusion of unpublished, negative studies, as the exclusion of these studies results in an asymmetrical funnel plot. This plot shows a slight predominance of data points from large studies below the horizontal line (representing the effect size), thus indicating an opposite effect of publication bias, as these studies have negative results (fig 5). Furthermore, the distribution of the funnel plot is fairly symmetrical, thus suggesting there is no publication bias. Finally, the $\mathrm{p}$ value for the Egger test was not significant $(p=0.12)$, therefore indicating that the results of this meta-analysis are not the result of publication bias.

\section{Meta-analysis results for ECT trials}

For the meta-analysis of the effects of ECT on motor function in PD patients, only five studies satisfied our inclusion criteria. The characteristics of these studies are described in tables 1 and 5. As only five studies were included in this meta-analysis, we only calculated the pooled effect size using 


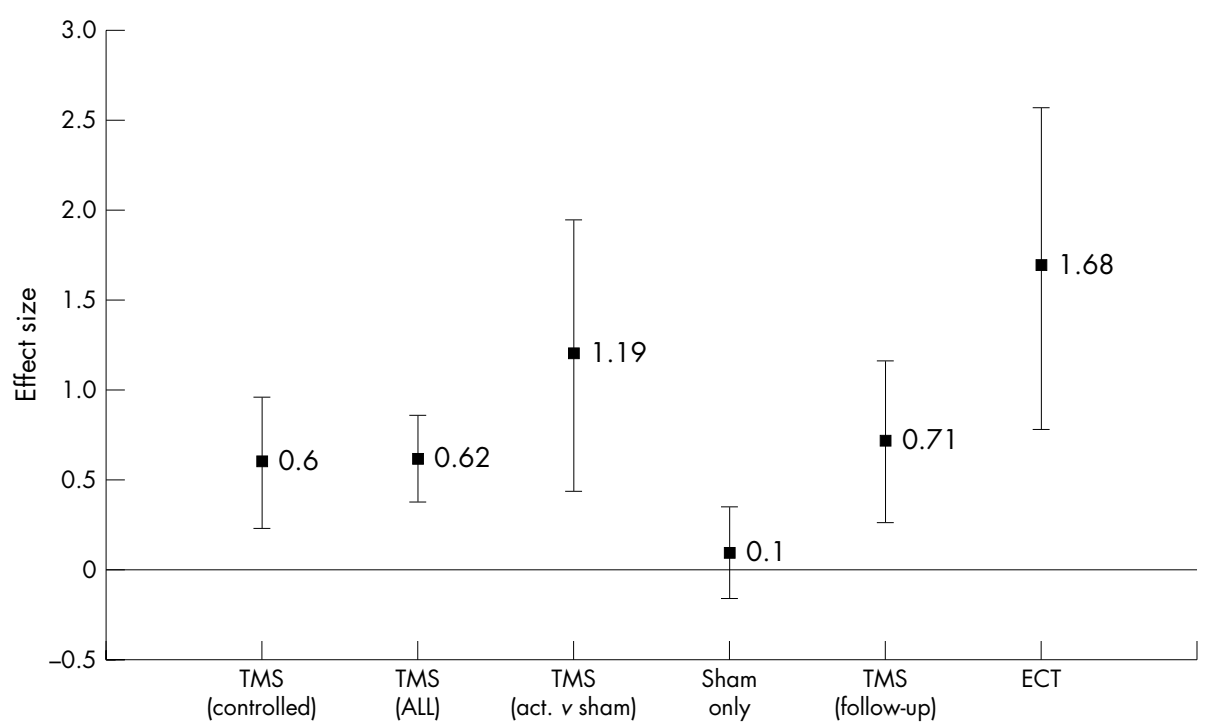

Figure 2 Effect sizes representations (filled squares) and their 95\% confidence intervals (lines) of the sub-analyses of the meta-analyses. TMS (controlled) indicates the TMS controlled studies only. TMS (ALL) indicates that the uncontrolled and controlled studies were pooled together. TMS (act. $v$ sham) indicates that the sham and active rTMS groups in the controlled trials were compared. Sham only indicates that only the sham group was analysed. TMS (follow-up) indicates that motor scores at the follow up (30 days or more) were compared to baseline. ECT is the pooled effect size for the ECT trials (five studies). A positive effect size indicates that the effect was larger in the post-treatment group, or favoured the active group.

the random and fixed effects models. This analysis showed a pooled effect size from the random effects model of $1.68(95 \%$ CI: $0.79,2.57$ ) (fig 2) and from the fixed effects model of 1.55 (95\% CI: 1.07, 2.03). The test for heterogeneity confirmed that there was a significant heterogeneity across the studies (Q4, $\left.\chi^{2}=12.95, \mathrm{p}=0.012\right)$. Because of the small number of studies (five), further analysis, such as meta-regression and Egger's test, could not be performed. Therefore, we could not systematically assess further heterogeneity and publication bias for this analysis, and thus the results of ECT trials should be interpreted with caution.

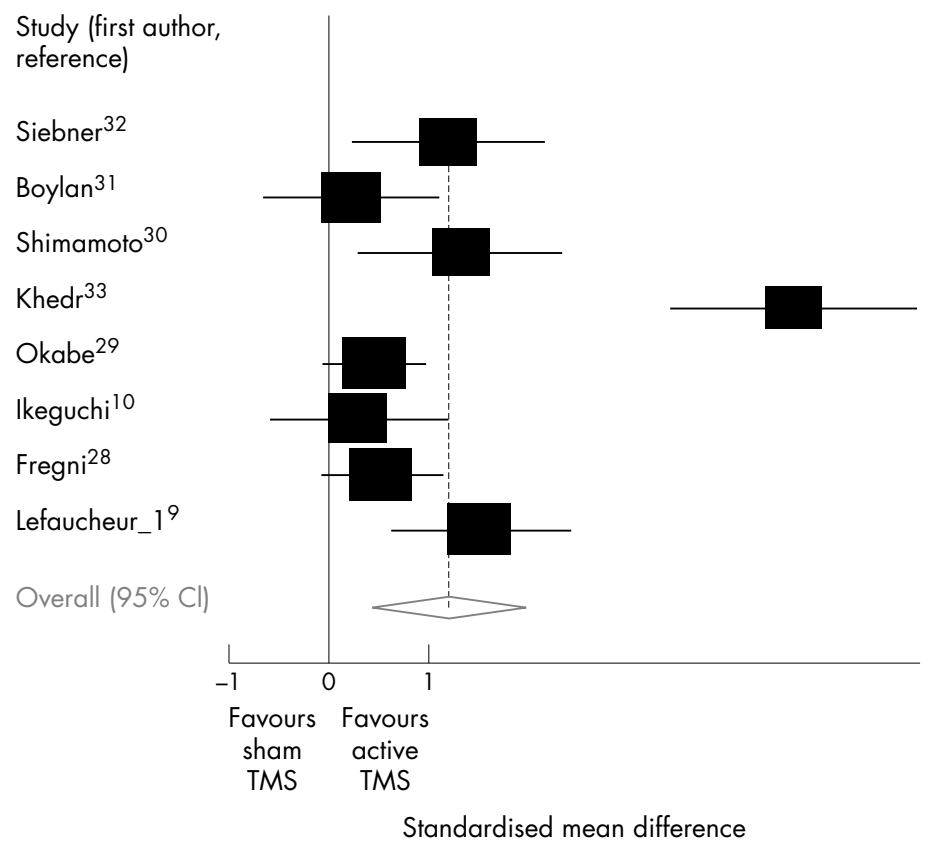

\section{DISCUSSION}

The results of this meta-analysis support the hypothesis that non-invasive brain stimulation (TMS and ECT) can be effective in improving motor symptoms in patients with PD. The analysis of TMS studies showed that this result is consistent across controlled and uncontrolled trials, but the effect is modest. Furthermore, we show evidence against a publication bias or significant heterogeneity, and demonstrate that the result remains robust after excluding any single study. Although we showed that the effects of ECT are significant and, indeed, had a larger effect size when

Figure 3 Effect sizes (standardised mean difference of the scores of the change in motor UPDRS from baseline to after treatment between the active and placebo group) from the random effects model. A positive effect indicates an improvement in motor function in the active group compared to the placebo group. Effect sizes are Cohen d (standardised mean difference), error bars represent the $95 \%$ confidence interval. 
Table 2 TMS study characteristics

\begin{tabular}{|c|c|c|c|c|c|c|c|c|}
\hline Author & Year & Patients* & Frequency & Pulses & Intensity & Coil & Sessions & Site \\
\hline Mally ${ }^{34}$ & 1999 & 10 & 1 & $30 \S$ & $0.2 \mathrm{MT}$ & C & 10 & Vertex \\
\hline Mally_1 ${ }^{7}$ & 1999 & 12 & 1 & 30 & $0.34 \mathrm{~T}$ & C & 10 & Vertex \\
\hline Mally_2 & 1999 & 12 & 1 & 30 & $0.34 \mathrm{~T}$ & C & 14 & Vertex \\
\hline Mally_37 & 1999 & 12 & 1 & 30 & $0.57 \mathrm{~T}$ & C & 10 & Vertex \\
\hline Mally_4 $4^{7}$ & 1999 & 12 & 1 & 30 & $0.80 \mathrm{~T}$ & C & 14 & Vertex \\
\hline Tergau ${ }^{35}$ & 1999 & 7 & $1,5,10,20$ & 1000 & $90 \%$ MT & $\mathrm{C}$ & 4 & NA \\
\hline Siebner ${ }^{32}$ & 2000 & 10 & 5 & 2250 & $90 \%$ MT & F8 & 1 & MI \\
\hline Boylan $^{31}$ & 2001 & 10 & 10 & 2000 & $110 \% \mathrm{MT}$ & F8 & 1 & SMA \\
\hline de Groot_1 ${ }^{8}$ & 2001 & 9 & 5 & 2250 & $90 \% \mathrm{MT}$ & F8 & 1 & MI \\
\hline de Groot_2 ${ }^{8}$ & 2001 & 9 & 5 & 2250 & $90 \%$ MT & F8 & 1 & M1 \\
\hline Shimamoto ${ }^{30}$ & 2001 & 9 & 0.2 & 60 & $700 \mathrm{~V}$ & C & 8 & Frontal \\
\hline Dragasevic $^{36}$ & 2002 & 10 & 0.5 & 200 & $110 \% \mathrm{MT}$ & $\mathrm{C}$ & 10 & Prefrontal \\
\hline Ikeguchi $^{10}$ & 2003 & 10 & 0.2 & 60 & $70 \% \dagger$ & $\mathrm{C}$ & 6 & Prefrontal \\
\hline Khedr ${ }^{33}$ & 2003 & 19 & 5 & 2000 & $120 \%$ MT & F8 & 10 & M1 \\
\hline $\mathrm{Okabe}^{29}$ & 2003 & $85 \ddagger$ & 0.2 & 100 & $110 \% \mathrm{MT}$ & C & 8 & Vertex \\
\hline Fregni ${ }^{28}$ & 2004 & 21 & 15 & 3000 & $110 \% \mathrm{MT}$ & F8 & 10 & Prefrontal \\
\hline Lefaucheur_19 & 2004 & 12 & 0.5 & 600 & $80 \% \mathrm{MT}$ & F8 & 1 & MI \\
\hline Lefaucheur_29 & 2004 & 12 & 10 & 2000 & $80 \%$ MT & F8 & 1 & MI \\
\hline
\end{tabular}

compared to TMS, the small number of trials limits our ability to draw any definite conclusion about this technique in PD patients.

\section{Negative and positive effect in rTMS trials}

Although this meta-analysis shows a favourable effect of TMS on motor function in PD, a positive effect was not observed in every trial. One of the reasons may be the small sample size of these negative studies. In this scenario, the meta-analysis technique is a valuable method to combine the data from small studies in order to provide a conclusion based on an analysis with better power. However, two studie $^{28}{ }^{29}$ with relatively large sample sizes showed negative results. One explanation for this contradiction might be the interaction of antiparkinson drugs with TMS, as these studies assessed the motor UPDRS after the use of levodopa ("on" state). This medication might mask the effects of TMS due to a ceiling effect. Therefore, assessment of patients in the "off" state may provide a more sensitive measure of the benefit of TMS. An alternative explanation is that the variability of the results stems from the wide range of TMS parameters and patient selection criteria used in these studies, that is, the optimal TMS parameters might vary depending on disease duration and severity. Although the meta-regression results failed to show that TMS parameters could significantly account for the variability across studies in motor improvement, the interaction term (TMS parameters versus patient characteristics) was not analysed because of lack of power for this type of test.

An important consideration is the low number of pulses and intensity in some of these trials, such as the studies of Mally and Stone ${ }^{7}$ (30 pulses twice a day, intensity of $20 \%$ of motor threshold (MT)) and Shimamoto et al ${ }^{30}$ (60 pulses per day, intensity of $700 \mathrm{~V})$. One can argue that these parameters were too low to induce a biological effect. However, the number of sessions may influence the clinical effects of this technique (for example, in treatment for depression ${ }^{40}$ ), and therefore the application of rTMS over several sessions in these studies might explain their reported significant effects.

The site of stimulation appears to be critical for rTMS induced motor improvement, and a focal coil, such as a figure-of-eight coil, should provide the greatest precision in targeted stimulation. However, a significant correlation was not found between motor improvement and coil type. For instance five of the seven studies which used circular coils showed a significant motor improvement and the two studies which used figure-of-eight coils did not show any significant motor improvement induced by rTMS. It is likely that the degree of motor improvement depends on interactions between coil type and other parameters, such as frequency, intensity, and stimulation site.

\section{Non-invasive brain stimulation for PD}

TMS effects are primarily directed at surface cortical regions. Since the dopaminergic deficiency in PD is localised to the subcortical basal ganglia, the beneficial effects of rTMS on PD motor symptoms are necessarily somewhat indirect. Two pathophysiological mechanisms can be proposed to explain how cortically directed rTMS may improve PD symptoms: either (i) rTMS induces network changes that connect with and positively affect basal ganglia function or (ii) rTMS to cortical sites compensates for systematic abnormal changes in cortical function associated with PD. Indeed, in support of the former mechanism, rTMS might modulate cortical areas, such as the prefrontal cortex and primary motor cortex, which are substantially connected to both the striatum and the subthalamic nucleus ${ }^{41}$ via glutamatergic projection, and thus indirectly modulate the release of dopamine in the basal ganglia. ${ }^{42}$ Several TMS/functional imaging studies have demonstrated the effects of rTMS on this neural network ${ }^{3} 4$ (although resolution of the images of the basal ganglia in

Table 3 Pooled weighted effect size and mean difference

\begin{tabular}{lllll}
\hline & $\begin{array}{l}\text { Random } \\
\text { effects model }\end{array}$ & $\mathbf{9 5 \% ~ C l}$ & $\begin{array}{l}\text { Fixed } \\
\text { effects model }\end{array}$ & $\begin{array}{l}\text { Q statistic, } \\
\mathbf{p} \text { value }\end{array}$ \\
\hline Pooled weighted mean difference (all studies) & 5.90 & $3.36,8.44$ & 4.15 & $2.79,5.50$ \\
Pooled weighted effect size* (all studies) & 0.62 & $0.38,0.85$ & 0.59 & 0.001 \\
Pooled weighted effect size* (controlled studies) & 0.60 & $0.24,0.96$ & 0.56 & $0.70,0.78$ \\
\hline
\end{tabular}

*Effect size: standardised mean difference. The mean difference was calculated using the change from pre- to post-treatment UPDRS for the active group. $95 \%$ Cl, $95 \%$ confidence interval. 
Table 4 Meta-regression results

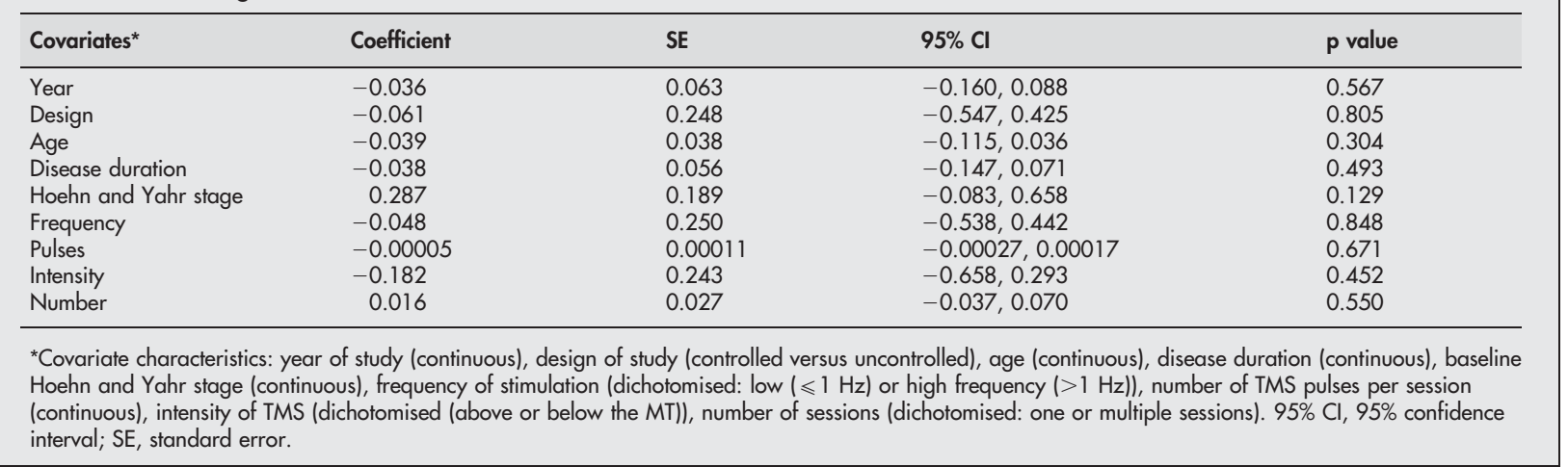

most of these studies can be limited) and an increase of dopamine in basal ganglia after rTMS of the frontal lobe. ${ }^{43} 44$

In support of the latter mechanism, functional imaging and TMS studies of PD subjects have demonstrated altered cortical physiology in basal ganglia connected areas such as the supplementary motor area, dorsolateral prefrontal cortex, and primary motor cortex,,$^{41} 45$ characterised by excessive corticospinal output at rest and reduced intracortical inhibition. Because a given motor task is associated with suppression of competing motor networks, these cortical changes in PD patients might avoid this suppression and therefore decrease the performance of the motor system, resulting in symptoms such as tonic contractions and rigidity. ${ }^{41}$ Therefore, rTMS may serve to compensate for the standard basal ganglia model of underactive pallido-thalamo-cortical drive ${ }^{817}$ and to modulate cortical excitability to correct for (pseudonormalise) known or suspected abnormalities in cortical excitability associated with PD. ${ }^{19} 34$ Although these mechanisms of action are based on several studies which have attempted to elucidate the pathophysiology of motor disturbance in PD, they remain unproven, and further investigations are required.

Likewise, the putative mechanism of action of ECT in PD is still unknown. One can conjecture that the effects of ECT on the brain are similar to those following rTMS, but the effects might be amplified as the electric current induced by ECT spreads to a larger area when compared to TMS and induces a greater voltage. ${ }^{47}$ Although several mechanisms to explain

Study (first author,

reference)

Mally

Mally_17

Mally_27

Mally_37

Mally_4 $4^{7}$

Tergau 35

Siebner ${ }^{32}$

Boylan 31

Shimamoto 30

de Groot_18

de Groot_28

Dragasevic ${ }^{36}$

Khedr ${ }^{33}$

Okabe $^{29}$

Ikeguchi 10

Fregni28

Lefaucheur_1 $^{9}$

Lefaucheur_29

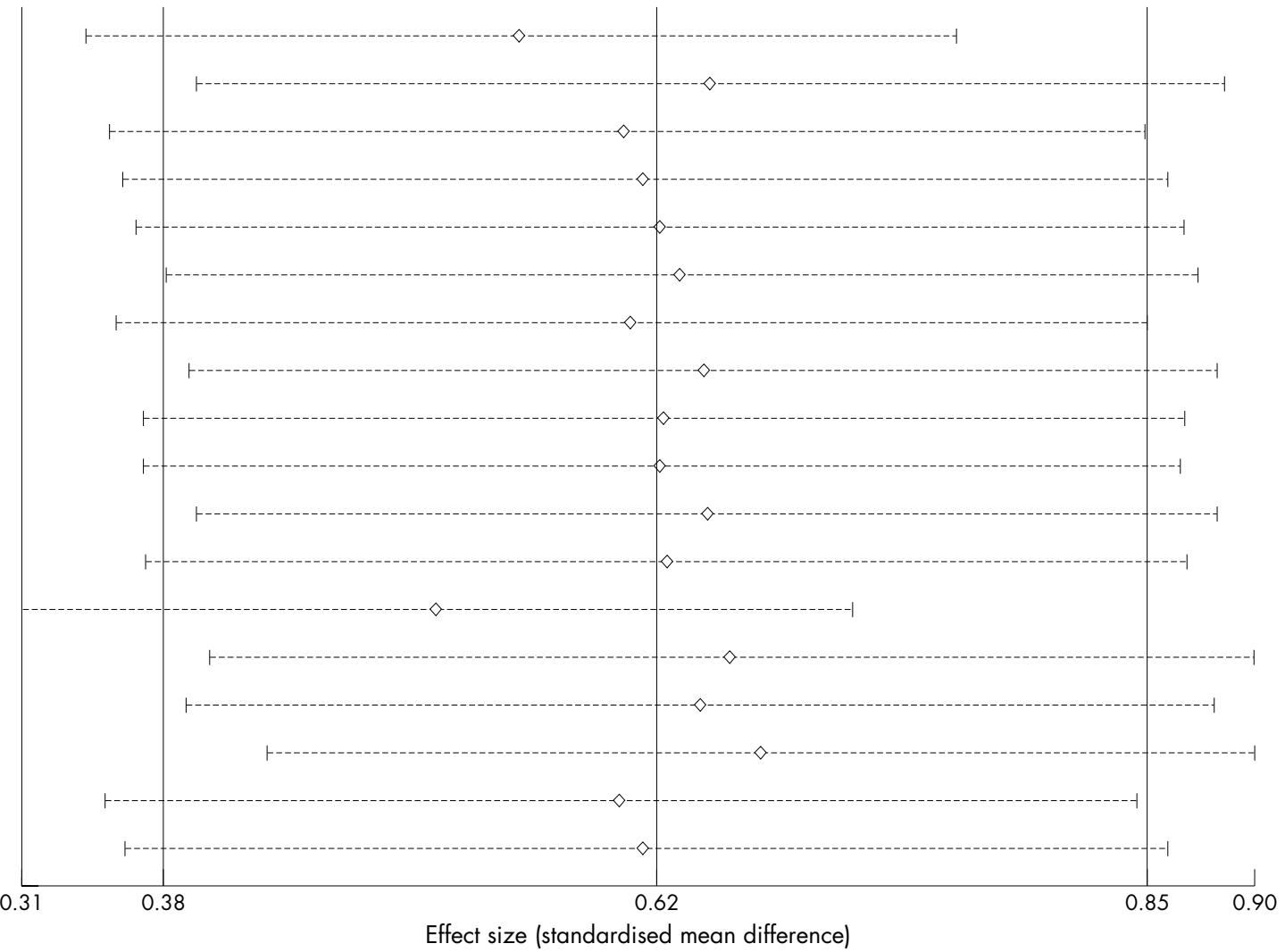

Figure 4 Assessment of the individual influence of each study. The change in the overall effect size and $95 \%$ confidence intervals for the meta-analysis after eliminating the indicated study is shown. Effect sizes are Cohen d (standardised mean difference), error bars represent the $95 \%$ confidence interval. 


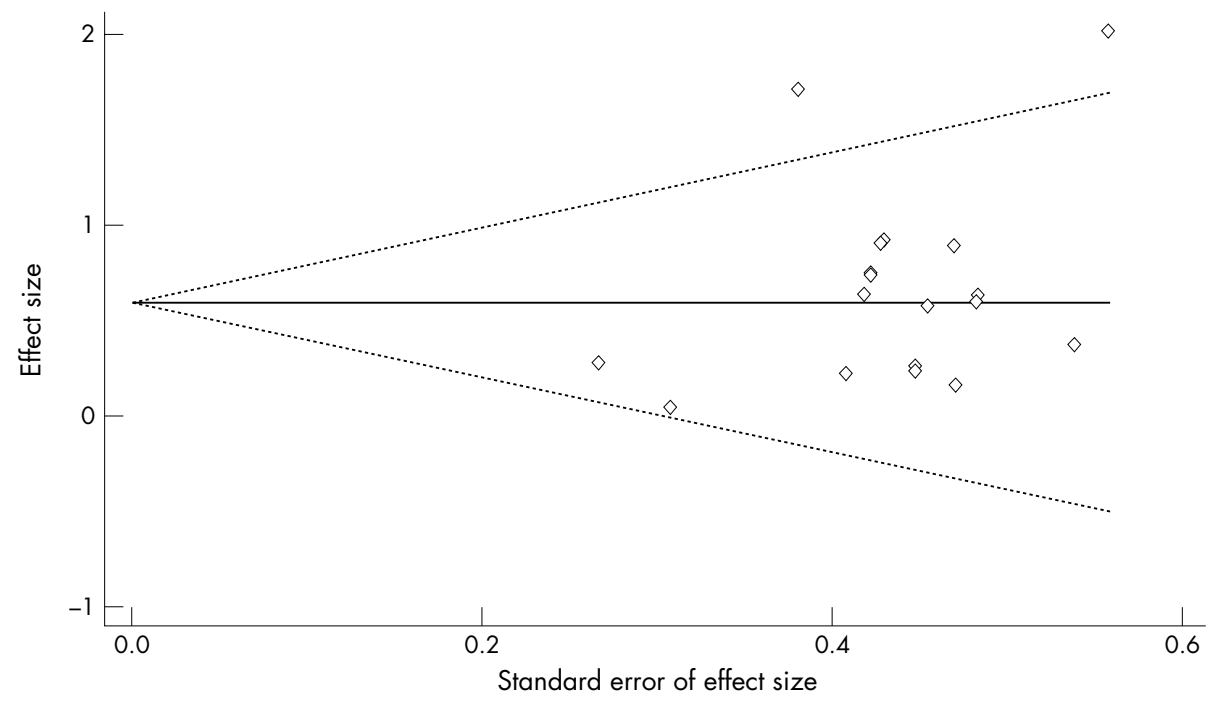

Figure 5 Funnel plot (publication bias assessment) of the effect sizes (Cohen d) according to their standard errors. The horizontal solid line is drawn at the pooled effect size, and angled lines represent the expected $95 \%$ confidence interval for a given standard error, assuming no between study heterogeneity.

ECT effects on motor function in PD have been proposed (for example, enhancement of dopaminergic receptors, ${ }^{48}$ improvement of depression, ${ }^{49}$ disruption of the blood-brain barrier $\left.{ }^{50}\right)$, convincing evidence to support these assumptions is lacking. Finally, the role or confound of the seizure which is always associated with ECT, as opposed to rTMS, remains unclear.

\section{Clinical implications}

The results of this meta-analysis suggest that rTMS might be an effective treatment for patients with PD, highlighting the need for additional more definitive clinical studies in PD patients. For a treatment to be considered clinically useful in PD patients, it should fulfil the following criteria: (i) the therapy has to have a long lasting effect (at least hours or days); (ii) the motor improvement has to be clinically meaningful; and (iii) the clinical benefits of a new therapy should outweigh its side effects. Regarding the long lasting effect, only six studies identified in this meta-analysis investigated the long lasting effects of TMS in PD patients. The pooled analysis of these six studies suggested that the effect of one or a few sessions of TMS can last at least 30 days after the end of the treatment. Indeed, a long lasting effect of rTMS has been demonstrated previously in patients with major depression, schizophrenia, and stroke and aphasia. ${ }^{51} 52$ Furthermore, recent studies suggest that even longer term benefits with maintenance TMS treatments are indeed possible and effective for the treatment of depression ${ }^{53}$ and $\mathrm{PD}^{54}$

Another important issue is whether the effects of TMS on motor function are clinically relevant, as a statistically significant difference between two treatments (or before

\begin{tabular}{|lllll|}
\hline \multicolumn{4}{l}{ Table 5 } & \multicolumn{4}{l}{ ECT study characteristics } \\
\hline Author & Year & Patients & ECT parameters & Sessions \\
\hline Balldin $^{37}$ & 1981 & 9 & Bilateral & $4-8$ \\
Andersen $^{6}$ & 1987 & 11 & Bilateral & NA \\
Douyon $^{38}$ & 1989 & 7 & Bilateral & 7 \\
Fall $^{5}$ & 1995 & 16 & Unilateral & $4-9$ \\
Fall $^{39}$ & 2000 & 6 & Unilateral & $6-7$ \\
\hline NA, not available. & & & \\
\hline
\end{tabular}

and after treatment) does not necessarily equal clinical importance. To analyse this effect, we also report the mean difference (rather than the standardised mean difference) for the TMS studies (as these studies utilised the same scale, that is the motor section of the UPDRS). The pooled mean difference was 5.90 (95\% CI: 3.36, 8.44) points on the UPDRS scale (this represents an improvement of more than $20 \%$ in motor function compared to the baseline UPDRS). The possibility of a placebo effect must be considered as well. Goetz et al reported a significant placebo effect in 17\% of PD patients submitted to a chronic new antiparkinsonian therapy. ${ }^{55}$ However, the effect of rTMS on motor UPDRS scores remained significant even for the subset of studies that compared the active rTMS group to a placebo TMS group (fig 3). There also may be differences in the impact of the placebo effect between acute treatment (such as rTMS for one session) and chronic treatment (such as the use of a new drug for several months). For instance, Fregni et al showed no significant motor improvement after a single session of placebo rTMS compared to a levodopa challenge. ${ }^{56}$ Furthermore, studies in which several sessions of rTMS were administered, such as those reported by Mally et al and Khedr et al, have larger clinical effects (substantially larger than $20 \%) .{ }^{33}$ Finally, it is possible that optimisation of the TMS treatment protocol and patient selection could result in benefits of greater magnitude. However, it is premature to conclude that long term treatment with TMS might be as effective as treatment with levodopa. Although a retrospective study ${ }^{54}$ raised the possibility that rTMS combined with drugs can slow the development of PD, a proper clinical trial with an adequate sample size, methodology, and a long follow up comparing TMS and pharmacological treatment would be desirable.

It is important to consider the side effects of rTMS. In fact, rTMS is a technique associated with only a few, mild adverse events. ${ }^{57}$ Analysing the side effects of the studies included in this meta-analyses, four of these studies reported no side effects. $^{8-10}{ }^{33}$ Boylan et al reported that one out of 10 patients could not receive repetitive TMS as single pulse motor studies induced an exaggerated startle response and marked worsening of tremor. ${ }^{31}$ In the study by Dragasevic et al, ${ }^{36}$ although all the patients tolerated the treatment well, the authors reported that four patients complained of a light burning sensation over the scalp during stimulation and 
three patients had mild tension headache. Finally, Fregni et al reported mild, benign side effects such as mild headache, neck pain, a mild scalp burning sensation, and increase of salivation, which were more prevalent in the control group compared to the active rTMS group. ${ }^{28}$

The result of this meta-analysis opens up an avenue for the exploration of electrical stimulation. For example, studies are needed to assess the efficacy of new methods of brain stimulation in PD patients. Transcranial direct current stimulation is one of these therapies which might be valuable in PD. Recent studies have shown that this therapy can induce modulatory effects in the brain cortex similar to those induced by rTMS $^{58}$ Cortical (epidural) stimulation is another therapy which has been investigated for PD. A case report ${ }^{59}$ and animal study ${ }^{60}$ showed that epidural motor cortex stimulation may be a good approach to improve symptoms of PD and the benefits may be longer lasting than those following rTMS. In any case, even if the effects of non-invasive rTMS were to prove to be short lived, an rTMS study may be useful to assess the suitability of a given patient for more invasive, cortical stimulation. Extradural cortical stimulation has the advantage (compared to subdural cortical stimulation) of being minimally invasive (it needs only local anaesthesia to implant the electrodes and is associated with fewer post-operative complications, such as infection and haemorrhage). Future studies are needed to investigate and compare the efficacy of different types of motor cortex stimulation.

\section{Conclusion}

This meta-analysis shows that rTMS and ECT can produce statistically significant effects on motor function in PD. Although the results of this TMS meta-analysis are robust and stable (that is, not substantially altered by excluding any single study), its effect size was moderate. For ECT, although there was a relatively large and significant effect size, we considered the low number of studies to be a limiting factor, and therefore avoid any definite conclusions about this method of brain stimulation in PD. Furthermore, the results of this meta-analysis do not answer whether or not noninvasive brain stimulation would have a clinically meaningful benefit in PD patients. However, our findings encourage further larger and carefully designed clinical trials to assess the potential clinical value of rTMS for PD patients.

\section{ACKNOWLEDGEMENTS}

The authors would like to thank Steven D Freedman for advice, mentoring, and support; Munir Boodhwani for help with data analysis; and Professor Simin Liu and Emily Levitan, from the Department of Epidemiology of Harvard School of Public Health, for their comments and suggestions on an earlier version of the manuscript.

\section{Authors' affiliations}

F Fregni, A Pascual-Leone, Harvard Center for Noninvasive Brain Stimulation, Harvard Medical School and Beth Israel Deaconess Medical Center, Boston, MA, USA

D K Simon, Department of Neurology, Harvard Medical School and Beth Israel Deaconess Medical Center, Boston, MA, USA

A Wu, Department of Neurology, University of Southern California, Los Angeles, CA, USA

This work was supported by a grant from the Harvard Medical School Scholars in Clinical Science Program (NIH K30 HL04095-03) to FF and K24 RR018875 to APL

Competing interests: none declared

\section{REFERENCES}

1 Olanow CW, Watts RL, Koller WC. An algorithm (decision tree) for the management of Parkinson's disease (2001): treatment guidelines. Neurology 2001;56(11 Suppl 5):S1-88.
2 Boucai L, Cerquetti D, Merello M. Functional surgery for Parkinson's disease treatment: a structured analysis of a decade of published literature. Br J Neurosurg 2004;18(3):213-22.

3 Mottaghy FM, Krause BJ, Kemna $\sqcup$, et al. Modulation of the neuronal circuitry subserving working memory in healthy human subjects by repetitive transcranial magnetic stimulation. Neurosci Lett 2000;280(3):167-70.

4 Paus T, Castro-Alamancos MA, Petrides M. Cortico-cortical connectivity of the human mid-dorsolateral frontal cortex and its modulation by repetitive transcranial magnetic stimulation. Eur J Neurosci 2001;14(8):1405-11.

5 Fall PA, Ekman R, Granerus AK, et al. ECT in Parkinson's disease. Changes in motor symptoms, monoamine metabolites and neuropeptides. J Neural Transm Park Dis Dement Sect 1995; 10(2-3):129-40.

6 Andersen K, Balldin J, Gotffries CG, et al. A double-blind evaluation of electroconvulsive therapy in Parkinson's disease with "on-off" phenomena. Acta Neurol Scand 1987;76(3):191-9.

7 Mally J, Stone TW. Therapeutic and "dose-dependent" effect of repetitive microelectroshock induced by transcranial magnetic stimulation in Parkinson's disease. J Neurosci Res 1999;57(6):935-40.

8 de Groot M, Hermann W, Steffen J, et al. [Contralateral and ipsilateral repetitive transcranial magnetic stimulation in Parkinson patients]. Nervenarzt 2001;72(12):932-8.

9 Lefaucheur JP, Drouot X, Von Raison F, et al. Improvement of motor performance and modulation of cortical excitability by repetitive transcranial magnetic stimulation of the motor cortex in Parkinson's disease. Clin Neurophysiol 2004;115(11):2530-41.

10 Ikeguchi M, Touge T, Nishiyama Y, et al. Effects of successive repetitive transcranial magnetic stimulation on motor performances and brain perfusion in idiopathic Parkinson's disease. J Neurol Sci 2003;209(1-2):41-6.

11 Egger M, Smith GD, Phillips AN. Meta-analysis: principles and procedures. BMJ 1997;315(7121):1533-7.

12 Egger M, Davey Smith G, Schneider M, et al. Bias in meta-analysis detected by a simple, graphical test. BMJ 1997;315(7109):629-34.

13 Pascual-Leone A, Valls-Sole J, Brasil-Neto JP, et al. Akinesia in Parkinson's disease. I. Shortening of simple reaction time with focal, single-pulse transcranial magnetic stimulation. Neurology 1994;44(5):884-91.

14 Pascual-Leone A, Valls-Sole J, Brasil-Neto JP, et al. Akinesia in Parkinson's disease. II. Effects of subthreshold repetitive transcranial motor cortex stimulation. Neurology 1994;44(5):892-8.

15 Pascual-Leone A, Alonso A, Pascual-Leone Pascual A. Lasting beneficial effect of rapid-rate transcranial magnetic stimulation on slowness in Parkinson's disease (abstract). Neurology 1995;45(Suppl 4):A315.

16 Cunnington $R$, lansek R, Thickbroom GW, et al. Effects of magnetic stimulation over supplementary motor area on movement in Parkinson's disease. Brain 1996; 119(Pt 3):815-22.

17 Ghabra MB, Hallett M, Wassermann EM. Simultaneous repetitive transcranial magnetic stimulation does not speed fine movement in PD. Neurology 1999;52(4):768-70

18 Valls-Sole J, Pascual-Leone A, Brasil-Neto JP, et al. Abnormal facilitation of the response to transcranial magnetic stimulation in patients with Parkinson's disease. Neurology 1994;44(4):735-41.

19 Siebner HR, Mentschel C, Aver C, et al. Repetitive transcranial magnetic stimulation has a beneficial effect on bradykinesia in Parkinson's disease. Neuroreport 1999; 10(3):589-94.

20 Ellaway PH, Davey NJ, Maskill DW, et al. The relation between bradykinesia and excitability of the motor cortex assessed using transcranial magnetic stimulation in normal and parkinsonian subjects. Electroencephalogr Clin Neurophysiol 1995;97(3): 169-78.

21 Bornke C, Schulte T, Przuntek H, et al. Clinical effects of repetitive transcranial magnetic stimulation versus acute levodopa challenge in Parkinson's disease. J Neural Transm Suppl 2004;68:61-7.

22 Fall PA, Granerus AK. Maintenance ECT in Parkinson's disease. J Neural Transm 1999;106(7-8):737-41.

23 Zervas IM, Fink M. ECT and delirium in Parkinson's disease. Am J Psychiatry 1992; 149(12):1758.

24 Pridmore S, Lowrie A, Holmes G, et al. ECT in Parkinson's disease: neuropsychological response. Convuls Ther 1996;12(4):257-9.

25 Ward C, Stern GM, Pratt RT, et al. Electroconvulsive therapy in Parkinsonian patients with the "on-off" syndrome. J Neural Transm 1980;49(1-2):133-5.

26 Pridmore S, Pollard C. Electroconvulsive therapy in Parkinson's disease: 30 month follow up. J Neurol Neurosurg Psychiatry 1996;60(6):693.

27 Balldin J, Eden S, Granerus AK, et al. Electroconvulsive therapy in Parkinson's syndrome with "on-off" phenomenon. J Neural Transm 1980;47(1):11-21.

28 Fregni F, Santos CM, Myczkowski ML, et al. Repetitive transcranial magnetic stimulation is as effective as fluoxetine in the treatment of depression in patients with Parkinson's disease. J Neurol Neurosurg Psychiatry 2004;75(8): 1171-4

29 Okabe S, Ugawa Y, Kanazawa I. 0.2- $\mathrm{Hz}$ repetitive transcranial magnetic stimulation has no add-on effects as compared to a realistic sham stimulation in Parkinson's disease. Mov Disord 2003;18(4):382-8.

30 Shimamoto H, Takasaki K, Shigemori M, et al. Therapeutic effect and mechanism of repetitive transcranial magnetic stimulation in Parkinson's disease. J Neurol 2001;248(Suppl 3):III48-52.

31 Boylan LS, Pullman SL, Lisanby SH, et al. Repetitive transcranial magnetic stimulation to SMA worsens complex movements in Parkinson's disease. Clin Neurophysiol 2001;112(2):259-64

32 Siebner HR, Rossmeier C, Mentschel C, et al. Short-term motor improvement after sub-threshold $5-\mathrm{Hz}$ repetitive transcranial magnetic stimulation of the primary motor hand area in Parkinson's disease. J Neurol Sci $2000 ; 178(2): 91-4$ 
33 Khedr EM, Farweez HM, Islam $\mathrm{H}$. Therapeutic effect of repetitive transcranial magnetic stimulation on motor function in Parkinson's disease patients. Eur J Neurol 2003; 10(5):567-72.

34 Mally J, Stone TW. Improvement in Parkinsonian symptoms after repetitive transcranial magnetic stimulation. J Neurol Sci 1999; 162(2): 179-84.

35 Tergau F, Wassermann EM, Paulus W, et al. Lack of clinical improvement in patients with Parkinson's disease after low and high frequency repetitive transcranial magnetic stimulation. Electroencephalogr Clin Neurophysiol Suppl 1999;51:281-8.

36 Dragasevic N, Potrebic A, Damjanovic A, et al. Therapeutic efficacy of bilateral prefrontal slow repetitive transcranial magnetic stimulation in depressed patients with Parkinson's disease: an open study. Mov Disord 2002; 17(3):528-32

37 Balldin J, Granerus AK, Lindstedt G, et al. Predictors for improvement after electroconvulsive therapy in parkinsonian patients with on-off symptoms. J Neural Transm 1981;52(3):199-211.

38 Douyon R, Serby M, Klutchko B, et al. ECT and Parkinson's disease revisited: a "naturalistic" study. Am J Psychiatry 1989;146(11):1451-5.

39 Fall PA, Ekberg S, Granerus AK, et al. ECT in Parkinson's disease - dopamine transporter visualised by [123I]-beta-CIT SPECT. J Neural Transm 2000; 107(8-9):997-1008

40 Rumi DO, Gattaz WF, Rigonatti SP, et al. Transcranial magnetic stimulation accelerates the antidepressant effect of amitriptyline in severe depression: a double-blind placebo-controlled study. Biol Psychiatry 2005;57(2):162-6.

41 Lefaucheur JP. Motor cortex dysfunction revealed by cortical excitability studies in Parkinson's disease: influence of antiparkinsonian treatment and cortical stimulation. Clin Neurophysiol 2005;116(2):244-53.

42 Morari M, Marti M, Sbrenna S, et al. Reciprocal dopamine-glutamate modulation of release in the basal ganglia. Neurochem Int 1998;33(5):383-97.

43 Keck ME, Welt T, Muller MB, et al. Repetitive transcranial magnetic stimulation increases the release of dopamine in the mesolimbic and mesostriatal system. Neuropharmacology 2002;43(1):101-9.

44 Strafella AP, Paus T, Barrett J, et al. Repetitive transcranial magnetic stimulation of the human prefrontal cortex induces dopamine release in the caudate nucleus. J Neurosci 2001;21(15):RC157.

45 Catalan MJ, Ishii K, Honda M, et al. A PET study of sequential finger movements of varying length in patients with Parkinson's disease. Brain 1999; 122(P+ 3):483-95.
46 Sabatini U, Boulanouar K, Fabre N, et al. Cortical motor reorganization in akinetic patients with Parkinson's disease: a functional MRI study. Brain 2000; 123(Pt 2):394-403.

47 Lisanby SH, Belmaker RH. Animal models of the mechanisms of action of repetitive transcranial magnetic stimulation (RTMS): comparisons with electroconvulsive shock (ECS). Depress Anxiety 2000;12(3):178-87.

48 Fochtmann L. A mechanism for the efficacy of ECT in Parkinson's disease. Convuls Ther 1988;4(4):321-7

49 Kennedy R, Mittal D, O'Jile J. Electroconvulsive therapy in movement disorders: an update. J Neuropsychiatry Clin Neurosci 2003;15(4):407-21.

50 Bolwig TG, Hertz MM, Holm-Jensen J. Blood-brain barrier during electroshock seizures in the rat. Eur J Clin Invest 1977;7(2):95-100.

51 Naeser MA, Martin PI, Nicholas M, et al. Improved picture naming in chronic aphasia after TMS to part of right Broca's area: an open-protocol study. Brain Lang 2005;93(1):95-105.

52 Dannon PN, Dolberg OT, Schreiber S, et al. Three and six-month outcome following courses of either ECT or rTMS in a population of severely depressed individuals - preliminary report. Biol Psychiatry 2002;51(8):687-90.

53 Li X, Nahas Z, Anderson B, et al. Can left prefrontal rTMS be used as a maintenance treatment for bipolar depression? Depress Anxiety 2004;20(2):98-100

54 Mally J, Farkas R, Tothfalusi L, et al. Long-term follow-up study with repetitive transcranial magnetic stimulation (rTMS) in Parkinson's disease. Brain Res Bull 2004;64(3):259-63.

55 Goetz CG, Leurgans S, Raman R. Placebo-associated improvements in motor function: comparison of subjective and objective sections of the UPDRS in early Parkinson's disease. Mov Disord 2002;17(2):283-8.

56 Fregni F, Maia F, Boggio PS, et al. The placebo effect in Parkinson's disease patients: is this an acute effect? Mov Disord 2004;19/Suppl 9):S228.

57 Wassermann EM. Risk and safety of repetitive transcranial magnetic stimulation: report and suggested guidelines from the International Workshop on the Safety of Repetitive Transcranial Magnetic Stimulation, June 5-7, 1996. Electroencephalogr Clin Neurophysiol 1998;108(1):1-16.

58 Nitsche MA, Paulus W. Sustained excitability elevations induced by transcranial DC motor cortex stimulation in humans. Neurology 2001;57(10):1899-901

59 Canavero S, Paolotti R, Bonicalzi V, et al. Extrarlural motor cortex stimulation for advanced Parkinson disease. Report of two cases. J Neurosurg 2002;97(5):1208-11.

60 Drouot X, Oshino S, Jarraya B, et al. Functional recovery in a primate model of Parkinson's disease following motor cortex stimulation. Neuron 2004:44(5):769-78. 